\title{
Unilateral Visual loss Due to Methanol Intoxication: A Case Report
}

\author{
Hoorvash Faraji Dana ${ }^{1}$, Nasim Zamani ${ }^{2}$, Lida Shojaei Arani ${ }^{1}$, Ali Faraji ${ }^{3}$, and Kiumars \\ Bahmani $^{4}$ \\ ${ }^{1}$ Shahid Beheshti University of Medical Sciences School of Medicine \\ ${ }^{2}$ Loghman Hakim Hospital \\ ${ }^{3}$ Alborz University of Medical Sciences \\ ${ }^{4}$ Shahid Beheshti University of Medical Sciences
}

January 28, 2021

\begin{abstract}
A 45-year-old man with bilateral blurred vision referred to toxicology department, after treatment he suffered from vision loss in left eye while his right eye was intact. Visual impairment due to methanol poisoning usually occurs bilaterally, but in our case was interestingly unilateral.
\end{abstract}

\section{Unilateral Visual loss Due to Methanol Intoxication: A Case Report}

Hoorvash Faraji Dana ${ }^{1}$, Nasim Zamani ${ }^{2}$, Lida Shojaei Arani ${ }^{1}$, Ali Faraji ${ }^{3}$, Kiumars Bahmani ${ }^{4 *}$

1. MD, Clinical Toxicology Fellowship, Department of Clinical Toxicology, Loghman-Hakim Hospital Poison Center, Faculty of Medicine, Shahid Beheshti University of Medical Sciences, Tehran, Iran

2. Department of clinical toxicology, Loghman Hakim Hospital, shahid Beheshti University of Medical Sciences, Tehran, Iran

3. Medical Student, Student Research Committee, School of Medicine, Alborz University of Medical Sciences, Karaj, Alborz, Iran

4. PhD student of Toxicology, Department of Pharmacology and Toxicology, School of Pharmacy, Shahid Beheshti University of Medical Science, Tehran, Iran

Corresponding author email: Kiumarsbahmani@yahoo.com

\section{Key Clinical Message:}

A 45-year-old man with bilateral blurred vision referred to toxicology department, after treatment he suffered from vision loss in left eye while his right eye was intact. Visual impairment due to methanol poisoning usually occurs bilaterally, but in our case was interestingly unilateral.

\section{Keywords:}

Methanol Intoxication, Unilateral Blindness

\section{Introduction}

Methanol is a toxic alcohol which is metabolized in the body to formic acid and causes severe intoxication. Methanol poisoning is very dangerous because it can cause severe visual impairment (including irreversible bilateral blindness), metabolic acidosis, permanent neurological dysfunction and even death ${ }^{1}$. Irreversible visual loss may occur within hours to days after ingestion. Treatment regimens are aimed at controlling 
acid-base balance, removing the toxic agent by hemodialysis, or using ethanol solution as a competitive drug ${ }^{2}$. In this study we report a case of unilateral visual loss due to methanol intoxication.

\section{Case Presentation:}

A 45-year-old man referred to toxicology department of Loghman Hakim Hospital complaining of bilateral blurred vision, vomiting, nausea. He was oriented and he had drunk about $500 \mathrm{cc}$ alcohol two days ago and was suffering from blurred vision since yesterday.

Laboratory blood tests was as following (Table 1):

Patient's history, clinical sings and laboratory test results confirmed methanol toxicity so 4 hours of emergency hemodialysis performed for him, alcohol 96 140cc and 560cc D/W 5\% administered during hemodialysis, we also prescribed erythropoietin (Eprex 10000U) SC for 3 days, Methylprednisolone 250mg IV for 6 hours for 3 days, Amp folinic acid 60mg for 3 hours, 3 vial Na/HCO3 IV stat. VBG results after hemodialysis showed: PH: 7.54, PCO2:48.4, HCO3:23.7 So metabolic acidosis and right eye's vision was corrected but not the left eyes. Considering patient's condition hemodialysis was done for the second time, but he still suffered from central field loss in the left eye then he was referred to ophthalmologist that reported: right eye's acuity $7 / 10$, left eye's acuity 3m, RADP positive, left eye's abducent movement and its color was disturbed and retinal examination was consistent with methanol intoxication. Erythropoietin (Eprex 10000U) for 3 days and Brimogan (Brimonidine and Timolol) TDS has been prescribed for left eye.

\section{Discussion:}

Methanol poisoning can cause vision loss with damage to the visual pathways ${ }^{3}$. Studies have shown that the myelin sheath of the retrolaminar optic nerve is selectively vulnerable to methanol poisoning due to its anatomical structure ${ }^{4,5}$. Accumulation of formic acid metabolites is the cause of retinal and optic nerve damage. Depending on the severity of the poisoning and the duration of exposure to formic acid, this damage to visual structures may cause permanent vision loss ${ }^{6}$. Visual impairment due to methanol poisoning usually occurs bilaterally, but rare cases of unilateral vision damage have also been reported in two studies ${ }^{6,7}$. which they stated may be due to anatomical or structural variation. Treatment maybe the reason of vision improvement and some studies have reported improvement in vision loss after poisoning treatment ${ }^{8}$, but the return of vision was not symmetrical in our patient's eyes.

In conclusion such abnormal case reports can help to raise awareness of the diversity of the sequela of methanol poisoning.

\section{Conflict of interest :}

The authors declare there is no conflict of interest.

\section{Author Contribution:}

Dr. Nasim Zamani was responsible for management of the patient, Dr. Hoorvash Faraji Dana was responsible for ordering the laboratory tests, and treated the patient. Dr. Lida Shojaei Arani was responsible for consultation and also patient management, Dr. Ali Faraji collected the data and drafting the case presentation section, Dr. Kiumars Bahmani drafting the manuscript and reviewed the literature.

\section{References:}

1. Doreen B, Eyu P, Okethwangu D, et al. Fatal methanol poisoning caused by drinking adulterated locally distilled alcohol: Wakiso District, Uganda, June 2017. 2020;2020.

2. ROTENSTREICH Y, ASSIA EI, KESLER AJBjoo. Late treatment of methanol blindness. 1997;81(5):415-415.

3. Yang C, Tsai W, Lirng JJE. Ocular manifestations and MRI findings in a case of methanol poisoning. $2005 ; 19(7): 806-809$. 
4. Sharpe J, Hostovsky M, Bilbao J, Rewcastle NJAJoO. Methanol Optic Neuropathy. A Histopathological Study. 1983;95(1):134.

5. Benton Jr C, Calhoun Jr FJT-AAoO, Ophthalmology OAAo, Otolaryngology. The ocular effects of methyl alcohol poisoning: report of a catastrophe involving three hundred and twenty persons. 1952;56(6):875.

6. Lu JJ, Kalimullah EA, Bryant SMJJomt. Unilateral blindness following acute methanol poisoning. 2010;6(4):459-460.

7. Brahmi N, Blel Y, Abidi N, et al. Methanol poisoning in Tunisia: report of 16 cases. 2007;45(6):717-720.

8. Sivilotti ML, Burns MJ, Aaron CK, McMartin KE, Brent J. Reversal of severe methanol-induced visual impairment: no evidence of retinal toxicity due to fomepizole. Journal of toxicology Clinical toxicology. 2001;39(6):627-631.

\begin{tabular}{llllll}
\hline Test & Result & Test & Result & Test & Result \\
\hline PH & 7.16 & PTT & 29.9 & Na & 141 \\
HCO3 & 9.2 & INR & 1.03 & K & 5.1 \\
PCO2 & 9.2 & FBS & 140 & Aspirin & Negative \\
AST & 27 & PT & 11.2 & Ethanol & Negative \\
ALT & 25 & BUN & 28 & Methanol & 12.2 \\
ALP & 273 & PTT & 29.9 & Acetaminophen & $<5$ \\
PT & 11.2 & Cr & 1.4 & & \\
\hline
\end{tabular}

Table 1. laboratory tests result 